\title{
Association of anthropometric measures of obesity and chronic kidney disease in elderly women
}

\author{
Andrzej Jaroszynski ${ }^{1}$, Tadeusz Dereziński², Anna Jaroszyńska ${ }^{3}$, Tomasz Zapolski, \\ Beata Wąsikowska ${ }^{2}$, Andrzej Wysokiński ${ }^{3}$, Arkadiusz Jawień ${ }^{4}$, Wojciech Załuska ${ }^{5}$, \\ Andrzej Horoch ${ }^{6}$ \\ ${ }^{1}$ Department of Family Medicine, Medical University of Lublin, Poland \\ ${ }^{2}$ Out-Patient Clinic Esculap Gniewkowo,Gniewkowo, Poland \\ ${ }^{3}$ Department of Cardiology, Medical University of Lublin, Poland \\ ${ }^{4}$ Department of Vascular Surgary and Angiology Nicolaus Copernicus University, Bydgoszcz, Poland \\ ${ }^{5}$ Department of Nephrology, Medical University of Lublin, Poland \\ ${ }^{6}$ Institute of Rural Health, Lublin, Poland
}

\begin{abstract}
Jaroszynski A, Dereziński T, Jaroszyńska A, Zapolski T, Wąsikowska B, Wysokiński A, Jawień A, Załuska W, Horoch A. Association of anthropometric measures of obesity and chronic kidney disease in elderly women. Ann Agric Environ Med. 2016; 23(4): 636-640. doi: $10.5604 / 12321966.1226859$
\end{abstract}

\section{Abstract}

Introduction and objective. Growing evidence suggests that obesity is an important contributor to the development of chronic kidney disease (CKD). The relationship between obesity and CKD is complex and not completely understood, and the best anthropometric index of obesity in predicting CKD is controversial. This study aimed to determine the best anthropometric index of obesity in predicting CKD in a population of elderly women.

Materials and methods. Anthropometric indexes of obesity including body mass index (BMI), waist circumference (WC), waist-to-height ratio (WheiR) and waist-to-hip-ratio (WHR), were obtained in 730 selected females. Biochemical measurements including blood glucose, lipid profile, and 2-h postprandial blood glucose were performed. GFR was estimated by using CKD-EPI equation.

Results. The prevalence of CKD stage $\geq 3$ was $12.2 \%$. Overweight and obesity was found in $50 \%$ and $36 \%$ of participants, respectively. Increased central fat distribution, as defined by WheiR, WC and WHR, was found in $89.6 \%, 91.7 \%$ and $89.4 \%$ individuals, respectively. Univariate linear regression analysis showed positive correlations between CKD and age $(p<0.001)$, BMI $(p<0.001)$, WC $(p<0.001)$, WHR $(p=0.007)$, WheiR $(p<0.001)$, diabetes $(p=0.002)$, as well as triglicerydes $(p=0.031)$, and negative correlation between CKD and HDL level $(p=0.017)$. Multivariable analysis demonstrated that hypertension, diabetes, WC and WheiR were independent predictors of CKD. The area under the receiver operating characteristics curve was best for WheiR (0.647), followed by WC (0.620), BMI (0.616), and WHR (0.532).

Conclusions. Abdominal obesity is an important predictor of CKD. Of commonly used anthropometric parameters of obesity WheiR $\geq 0.6$ is particularly associated with CKD in elderly females.

\section{Key words}

obesity, chronic kidney disease, waist-to-height ratio, waist-to-hip ratio, body mass index, waist circumference

\section{INTRODUCTION}

Obesity is an escalating global health concern. In the last decade, the prevalence of obesity increased significantly at all ages in high-income countries $[1,2]$. Growing evidence suggests that obesity is a potentially important contributor to the development of chronic kidney disease (CKD). The relationship between obesity and CKD is complex and not completely understood. Obesity can influence CKD indirectly; however, a direct causality link between obesity and CKD appears highly likely. Obesity is recognized as a major risk factor for diabetes and hypertension, as well as cardiovascular disease, all of which are risk factors for CKD. Given that diabetes and hypertension account together for $\sim 70 \%$ of all cases of End Stage Renal Disease (ESRD), most of the increasing prevalence of CKD may be attributed to the sharp and parallel increase in obesity and diabetes during

Address for correspondence: Andrzej Jaroszynski, Department of Family Medicine Medical University of Lublin, Staszica 4-6, 20-081 Lublin, Poland

E-mail: jaroszynskiaj@interia.pl

Received: 02 October 2013; accepted: 14 February 2014 the same period $[1,3,4,5]$. An increasing body of evidence suggests, however, that obesity per se, even in the absence of diabetes, may attribute to renal damage. Adipose tissue is hormonally active and releases a large number of bioactive adipokine proteins that target numerous tissues and organs. Adipose tissue may affect the incidence and progression of CKD by oxidative stress, inflammation, insulin resistance, plasma lipids and coagulation abnormalities, and endothelial dysfunction $[4,5]$.

Given the epidemic rates of both obesity and CKD, and that obesity in potentially reversible, the ability to identify risk factors for developing CKD is crucial. However, the best anthropometric index of obesity in predicting CKD is controversial [5], especially in elderly women [5, 6, 7]. Moreover, there are ethnic differences affecting relations between CKD and anthropometric parametres [4, 5], and the association between CKD and obesity indexes have not been evaluated in the Polish population.

This study was designed to determine the best anthropometric index of obesity in predicting CKD in a population of elderly women. 


\section{MATERIALS AND METHOD}

Study participants. The study included female subjects, aged $65-80$, representing a population of women living in rural areas who reported for the screening for diabetes. The exclusion criteria were: neoplasma, NYHA class IV heart failure, severe liver damage, chronic inflammatory diseases, or other diseases affecting nutritional status. Excluded from the study were also subjects with altered TSH levels as well as those taking steroids. To verify chronicity, and thus to diagnose CKD, participants with eGFR $<60 \mathrm{ml} /$ min were included to the study only if a second creatinine measurement, at least 3 months before the first measurement, was available. All subjects gave written consent, and the studies were approved by the local Ethics Committee.

Basic data and biochemical measurements. Biochemical measurements, including blood glucose, lipid profile, and 2-h postprandial blood glucose, were performed. Blood was obtained after at least $10 \mathrm{~h}$ fasting. The following lipid parameters were measured by automated analysers: total cholesterol, high-density lipoprotein (HDL cholesterol) and triglycerides (TG). Low-density lipoprotein (LDL cholesterol) cholesterol was calculated using the Friedewald equation: LDL $(\mathrm{mg} / \mathrm{dl})=$ total cholesterol- HDL-(triglycerides/5). Smoking was defined as a history of smoking longer than two pack-years. Diabetes was defined as a history of treatment for diabetes or fasting glucose concentration $\geq 140 \mathrm{mg} / \mathrm{dl}$ or non-fasting glucose level $\geq 200 \mathrm{mg} / \mathrm{dl}$ [8]. Hypertension was defined as a history of hypertension that required antihypertensive therapy.

Glomerular filtration rate (GFR) estimation. GFR was estimated using the CKD-EPI equation [9]:

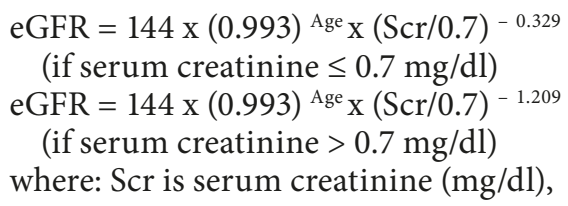

CKD was defined as an eGFR $<60 \mathrm{ml} / \mathrm{min}$ per $1.73 \mathrm{~m}^{2}$ [9].

Anthropometric measurements. Standard demographic information as well as anthropometric indexes of obesity, including body mass index (BMI), waist circumference (WC), waist-to-height ratio (WheiR) and waist-to-hip-ratio (WHR), were obtained in all participants. Height and weight were measured by professional health staff with the participants standing without shoes and heavy outer garments. BMI was calculated as weight divided by height squared $\left(\mathrm{kg} / \mathrm{m}^{2}\right)$. Waist circumference was measured at the level midway between the lower rib margin and the iliac crest with participants in a standing position without heavy outer garments and with emptied pockets, breathing out gently. Hip circumference was recorded as the maximum circumference over the buttocks. WHR and WheiR were consequently calculated by dividing the WC by the hip circumference and the height, respectively. Anthropometric measurements were categorized as follows: BMI was expressed as normal $\left(<25 \mathrm{~kg} / \mathrm{m}^{2}\right)$, overweight $(25<$ $\left.30 \mathrm{~kg} / \mathrm{m}^{2}\right)$ and obese $\left(>30 \mathrm{~kg} / \mathrm{m}^{2}\right)$; central fat distribution was defined as a WHR $\geq 0.85$; a WC $>80 \mathrm{~cm}$; a WheiR $>0.5[10]$.
Statistical analysis. Statistical analysis was carried out on an IBM PC using Statistica Version 10. Results were tested for normality by using the Kolmogorv-Smirnov test. When normally distributed, continuous variables were expressed as mean $\pm \mathrm{SD}$, and as median and range when non-normally distributed. Continuous data were compared using the Student t-test when normally distributed, and using MannWhitney U-test when non-normally distributed. Categorical data were expressed as frequencies and percentages and were compared using the $\chi^{2}$ test. Linear regression analysis was assessed using the Pearson correlation coefficient or Spearmans' rank correlation coefficient when appropriate. The area under the receiver operating characteristics curves (AUC) was to determine optimal cutoff points in predicting CKD. Multiple logistic regression analysis, with adjustment for age, was performed in order to establish the independent determinants of CKD. For all tests, a p-value $<0.05$ was considered statistically significant.

\section{RESULTS}

Of the total of 887 female initially identified, 148 subjects were not qualified due to exclusion criteria, and 9 participants with eGFR $<60 \mathrm{ml} / \mathrm{min}$ were excluded due to missing second creatinine measurements. The remaining 730 female subjects, aged $65-80$ years (mean $71.4 \pm 4.98$ ), entered the study.

Based on the eGFR calculated by CKD-EPI formula, the prevalence of CKD was $12.2 \%$. The distribution of eGFR is shown in Figure 1.

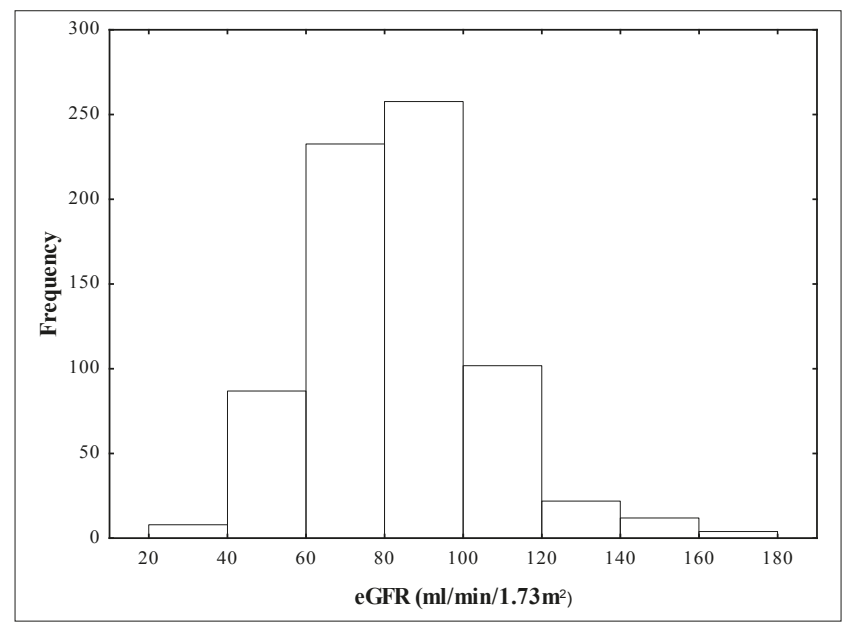

Figure 1. Distribution of eGFR

Baseline characteristics of all participants as well as split into two groups according to eGFR are shown in Table 1. Nearly $50 \%$ of the cohort were classified overweighr, and $36 \%$ were classified obese, whereas BMI was normal in 14.5\% participants. Increased central fat distribution as defined by WheiR, WC and WHR was found in $89.6 \%, 91.7 \%$ and $89.4 \%$ individuals, respectively.

Participants with CKD were older $(\mathrm{p}<0.001)$, had higher prevalence of either diabetes or hypertension $(\mathrm{p}<0.001$ in both cases), as well as smoking ( $\mathrm{p}=0.011)$, lower HDL concentration $(\mathrm{p}<0.001)$, and higher TG level $(\mathrm{p}<0.001)$. The serum creatinine, part of CKD-EPI formula, was also higher in subjects with CKD $(p<0.001)$. Participants with CKD also 
Table 1. Baseline characteristics of all participants split into two groups according to eGFR

\begin{tabular}{lcccc}
\hline & All $(\mathrm{n}=730)$ & $\begin{array}{c}\text { eGFR } \geq 60 \\
(\mathrm{n}=641)\end{array}$ & $\begin{array}{c}\text { eGFR }<60 \\
(\mathrm{n}=89)\end{array}$ & $\mathrm{p}$ \\
\hline Age (y) & $71.4 \pm 4.98$ & $70.5 \pm 4.72$ & $74.84 \pm 5.00$ & $<0.001$ \\
\hline Diabetes (\%) & 20.1 & 18.8 & 30.30 & $<0.001$ \\
\hline Hypertension (\%) & 39.6 & 36.8 & 59.6 & $<0.001$ \\
\hline Smoking (\%) & 18.2 & 17.6 & 22.5 & 0.011 \\
\hline Systolic blood pressure & $150.3 \pm 22.05$ & $150.3 \pm 21.921$ & $150.1 \pm 22.28$ & 0.925 \\
(mmHg) & & & & 0.124 \\
\hline $\begin{array}{l}\text { Diastolic blood pressure } \\
\text { (mmHg) }\end{array}$ & $83.67 \pm 10.47$ & $83.91 \pm 10.57$ & $81.92 \pm 9.37$ & 0.125 .0001 \\
\hline Body mass index (kg/m $\left.{ }^{2}\right)$ & $30.64 \pm 5.26$ & $30.42 \pm 5.28$ & $32.43 \pm 4.80$ & 0.001 \\
\hline Waist circumference (cm) & $98.02 \pm 12.50$ & $97.28 \pm 12.79$ & $103.2 \pm 8.95$ & $<0.001$ \\
\hline Waist-to-hip ratio & $0.870 \pm 0.085$ & $0.869 \pm 0.088$ & $0.878 \pm 0.055$ & 0.009 \\
\hline Waist-to-height ratio & $0.623 \pm 0.082$ & $0.619 \pm 0.084$ & $0.649 \pm 0.058$ & $<0.001$ \\
\hline Creatinine (mg/dl) & $0.735 \pm 0.190$ & $0.691 \pm 0.130$ & $1.15 \pm 0.52$ & $<0.001$ \\
\hline eGFR (ml/min) & $83.59 \pm 23.43$ & $87.57 \pm 21.51$ & $52.44 \pm 11.48$ & $<0.001$ \\
\hline Cholesterol (mg/dl) & $214.9 \pm 49.21$ & $215.1 \pm 48.76$ & $211.6 \pm 49.62$ & 0.536 \\
\hline LDL (mg/dl) & $124.4 \pm 45.05$ & $123.5 \pm 46.21$ & $127.7 \pm 44.87$ & 0.108 \\
\hline HDL (mg/d) & $65.88 \pm 17.48$ & $66.58 \pm 17.03$ & $60.14 \pm 15.01$ & $<0.001$ \\
\hline Triglycerides (mg/dl) & $127.7 \pm 65.11$ & $125.9 \pm 63.89$ & $141.3 \pm 67.03$ & $<0.001$ \\
\hline & & & & \\
\hline
\end{tabular}

Table 2. Associations between risk factors and chronic kidney disese

\begin{tabular}{cccc}
\hline & parameter & $\mathrm{r}$ & $\mathrm{p}$ \\
\hline & $\mathrm{age}$ & 0.263 & $<0.001$ \\
\cline { 2 - 4 } Chronic kidney disease & BMI & 0.127 & $<0.001$ \\
\cline { 2 - 4 } & WC & 0.175 & $<0.001$ \\
\cline { 2 - 4 } & WhR & 0.102 & 0.009 \\
\cline { 2 - 4 } & HDL & 0.182 & $<0.001$ \\
\cline { 2 - 4 } & TG & 0.086 & 0.021 \\
\cline { 2 - 4 } & smoking & 0.068 & 0.034 \\
\cline { 2 - 4 } & diabetes & 0.114 & 0.081 \\
\hline hypertension & 0.241 & $<0.003$ \\
\hline
\end{tabular}

BMI - body mass index; WC - waist circumference; WHR - waist-to-hip ratio; WheiR - Waist-toheight ratio; HDL - high-density lipoprotein cholesterol; TG - triglicerydes.

had higher values for BMI $(\mathrm{p}=0.001)$, WC $(\mathrm{p}<0.001)$, WHR $(\mathrm{p}=0.008)$, and WheiR $(\mathrm{p}<0.001)$.

Univariate linear regression analysis showed positive correlations between CKD and age $(\mathrm{p}<0.001), \mathrm{BMI}(\mathrm{p}<0.001)$, WC $(\mathrm{p}<0.001)$, WHR $(\mathrm{p}=0.007)$, WheiR $(\mathrm{p}<0.001)$, diabetes $(\mathrm{p}=0.002)$, as well as TG $(\mathrm{p}=0.031)$, and negative correlation between CKD and HDL concentration $(\mathrm{p}=0.017)$.

In order to indentify potential predictors of $\mathrm{CKD}$, logistic regression analysis with adjustment for age was performed. Multivariable analysis demonstrated that hypertension, diabetes, WC and WheiR were were independent predictors of CKD (Table 3).

The AUC was best for WheiR (0.647), followed by WC (0.620), BMI (0.616), and WHR (0.532) (Fig. 2). The optimal cut-off values were 0.60 for WheiR, 34 for BMI, 104 for WC, and 0.87 for WHR. At these cut-off points, the sensitivity and specificity were $68 \%$ and $60 \%$ for WheiR, $56 \%$ and $64 \%$ for WC, $51 \%$ and $61 \%$ for BMI, and $62 \%$ and $50 \%$ for WHR.
Table 3. Multivariable logistic regression analysis of association between chronic kidney disease and obesity indexes, as well as traditional risk factors with adjustment for age

\begin{tabular}{lccc}
\hline & OR & $95 \% \mathrm{Cl}$ & $\mathrm{p}$ \\
\hline diabetes & 2.1 & $1.64-2.82$ & $<0.001$ \\
\hline hypertension & 2.3 & $1.70-3.04$ & $<0.001$ \\
\hline smoking & 1.11 & $0.59-3.08$ & 0.393 \\
\hline $\mathrm{HDL}$ & 0.92 & $0.49-2.32$ & 0.632 \\
\hline TG & 1.07 & $0.74-2.03$ & 0.173 \\
\hline BMI & 1.19 & $0.61-2.97$ & 0.103 \\
\hline WC & 1.52 & $0.77-2.73$ & 0.096 \\
\hline WHR & 1.25 & $0.78-4.94$ & 0.515 \\
\hline WheiR & 1.38 & $0.89-1.74$ & 0.003 \\
\hline
\end{tabular}

Only baseline parameters with a $\mathrm{p}$ value $<0.10$ at the univariate analysis are shown.

$\mathrm{OR}$ - odds ratio; $\mathrm{Cl}$ - confidence interval; $\mathrm{BMI}$ - body mass index; WC - waist circumference; WHR - waist-to-hip ratio, WheiR - Waist-to-height $r$ atio; HDL - high-density lipoprotein cholesterol; TG - triglicerydes.

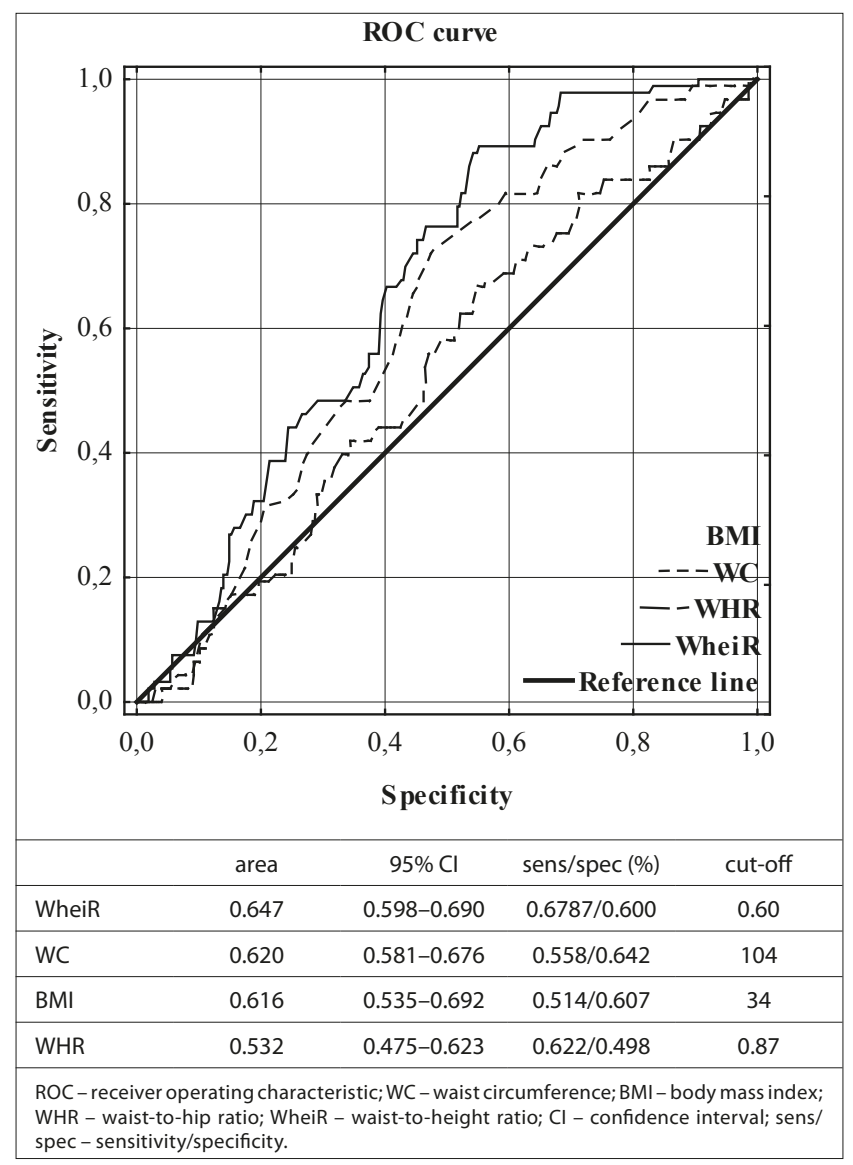

Figure 2. ROC curve of anthropometric indexes in predicting chronic kidney disease

\section{DISCUSSION}

The presented study provides evidence that abdominal obesity is an important predictor of CKD. Of the commonly used anthropometric parameters of obesity, WheiR $\geq 0.6$ is particularly associated with CKD in elderly females.

Growing evidence suggests that abdominal obesity is more strongly related to the presence and incidence of hypertension, diabetes, metabolic syndrome and cardiovascular disease mortality then peripheral fat $[11,12]$. Although WC, WHR, WheiR and BMI are often highly correlated, BMI provide 
data about entire body volume and mass, whereas WC and WHR, as well as WheiR, provide information about shape and fat distribution and are considered surrogate markers of central fat $[5,13,14,15,16]$. In the current study, WC and WheiR were independent predictors of CKD. This result is consistent with previous studies indicating that central fat is important risk factor for CKD $[11,15,17,18,19]$. Abdominal obesity may play a causal role in these associations by acting as an active endocrine organ. Central fat is associated with increased secretion of fatty acids, adipocytokines, hyperinsulinaemia, insulin resistance, hypertension, and atherogenic dyslipidemia $[14,20]$. In CKD patients, abdominal fat has been associated with inflammation, insulin resistance, dyslipidemia, oxidative stress, coronary artery calcification, cardiovascular events, and moratlity $[15,18,20]$.

A variety of methods can be used to assess abdominal obesity, including computed tomography, dual-energy $\mathrm{X}$-ray absorptiometry, and magnetic resonance imaging. However, clinically, these techniques are not practical. Given the clinical importance of the relationship between obesity and CKD, identification of simple anthropometric surrogate measures of obesity that could be used as screening tools to identify individuals at risk for CKD development is crucial. Although WheiR, WC as well as WHR correlate well with computed tomography assessment of intraabdominal fat [16], the best anthropometric index of obesity in predicting CKD is still controversial. It should be emphasized, however, that anthropometric parameters do not accurately reflect body fat and these parameters must be considered only as a screening tool [18].

In the presented study, the ROC analysis proved that measurements of central obesity, especially WheiR, showed higher discriminative ability for CKD then BMI. The 'rank' order for AUC was WheiR > WC > WHR > BMI. Recently published papers showed that WheiR was a superior tool for discriminating obesity-related cardiometabolic risk, as well as cardiovascular disease, compared with WC, WHR and BMI $[12,16]$; however, no data related to CKD. The current results are consistent with the results of Lin et al. [8] who showed that WheiR is particularly associated with CKD. Similarly, Tseng [21] found that WheiR was better associated with urinary albumin excretion than other anthropometric measures. However, both studie related to a Chineese population and it is well known that ethnic differences affect relations between CKD and anthropometric parametres $[4,5]$.

In this study, WheiR overperformed WC, WHR, as well as BMI in the detection of CKD. According to the authors' knowledge, the superiority of WheiR over other anthropometric measures in discriminating CKD risk have not been reported previously in a European population. The higher discriminative ability of WheiR at predicting CKD may probably result from the fact that WheiR has the advantage of better measurement of fat distribution in different ages and statures [22]. WC may over- or under-evaluate risk for tall or short individuals with similar WC [23]. The main limitation of the WHR is that both waist and hip can decrease with weight reduction and, as a consequence, changes in the ratio can frequently be small. Moreover, WHR increases in women with age which mostly reflects a reduction in fat deposits in the hips making this variable more difficult to interpret $[7,23]$. Results of the presented study failed to find that BMI is an idependent factor predisposing to CKD. Studies using BMI to evaluate the risk of CKD development have conflicting results. Some studies found that BMI is a strong risk factor for CKD [5]. Others, however, have not revealed a relationship between BMI and CKD [18, 24], or the relationship was found in males but not in females [6]. The disagreement between these results may be due to the differences in race, age, gender, or study design. Moreover, BMI has well known limitations: it assesses entire body mass and does not distinguish between central and peripheral fat, between subcutaneous and visceral fat, nor between weight from muscle, bone, fat or oedema $[5,18]$.

According to the WHO guidelines [10], WheiR $>0.5$ is the cut-off value characteristic for central fat distribution. In the current study it was found that WheiR value $>0.6$ is associated with CKD. This is in agreement with the results of previous studies which also suggested that a value above 0.6 indicates a substantially increased risk for cardiovascular disease [25].

In conclusion, this study has demonstrated that central obesity is an important, independent, and potentially preventable predictor of $\mathrm{CKD}$. WheiR $\geq 0.60$ seems to be a simple and reliable anthropometric predictor of CKD in elderly femles.

Limitations of the study. The main limitations of the present study is that relations between anthropometric parameters and changes in renal function were not estimated in time. Further long-term studies are needed to demonstrate the relationship between anthropometric parameters and renal function decline. The second limitation was relatively low number of patients. It would be also of interest to evaluate if the reduction in WheiR will reduce the risk of the development of CKD.

\section{REFERENCES}

1. Flegal K, Carroll M, Kit B, Ogden C. Prevalence of obesity and trends in the distribution of body mass index among US adults, 1999-2010. JAMA 2012; 307: 491-497.

2. Zatońska K, Janik-Koncewicz K, Regulska-Ilow B, Ilow R, Różańska D, Szuba A, et al. Prevalence of obesity - baseline assessment in the prospective cohort 'PONS' study. Ann Agric Environ Med. 2011; 18: $246-250$.

3. Levey A, de Jong P, Coresh J, El Nahas M, Astor B, Matsushita K, et al. The definition, classification, and prognosis of chronic kidney disease: a KDIGO Controversies Conference report. Kidney Int. 2011; 80: 17-28.

4. Ting S, Nair H, Ching I, Taheri S, Dasgupta I. Overweight, obesity and chronic kidney disease. Nephron Clin Pract. 2009; 112: 121-127.

5. Burton J, Gray L, Webb D, Davies M, Khunti K, Crasto W, et al. Association of anthropometric obesity measures with chronic kidney disease risk in a non-diabetic patient population. Nephrol Dial Transplant. 2012; 27: 1860-1866.

6. Iseki K, Ikemiya Y, Kinjo K, Inoue T, Iseki C, Takishita S. Body mass index and the risk of development of end-stage renal disease in a careened cohort. Kidney Int 2004; 65: 1870-1876.

7. Bartali B, Benvenuti E, Corsi A, Bandinelli S, Iorio A, Lauretani F, et al. Changes in anthropometric measures in man and women across the life-span: finding from the InCHIANTI study. Soz Praventivmed 2002; 47: 336-348.

8. Lin Ch, Chou C, Lin C, Huang C, Liu C, Lai S. Waist-to-height ratio is the best index of obesity in association with chronic kidney disease. Nutrition 2007; 23: 788-79.

9. Stevens L, Claybon M, Schmid C, Chen J, Horio M, Imai E, et al. Evaluation of the Chronic Kidney Disease Epidemiology Collaboration equation for estimating the glomerular filtration rate in multiple ethnicities. Kidney Int. 2011; 79, 555-562.

10. World Health Organisation. Waist circumference and waist-hip ratio: report of a WHO expert consultation, Geneva, 8-11 December 2008. World Health Organisation; 2011. 
11. Cordeiro A, Qureshi A, Lindholm B, Amparo F, Tito-Paladino-Filho A, Perini $M$, et al. Visceral fat and coronary artery calcification in patients with chronic kidney disease. Nephrol Dial Transplant 2013; doi:10.1093/ndt/gft250.

12. Gelber R, Gaziano J, Orav E, Manson J, Buring J, Kurth T. Measures of obesity and cardiovascular risk among men and women. J Am Coll Cardiol. 2008; 52: 605-615

13. Klein S, Allison D, Heymsfield S, Kelley D, Leibel R, Nonas C, et al. Waist circumference and cardiometabolic risk: a consensus statement from Shaping America's Health: Association for Weight Management and Obesity Prevention; NAASO, The Obesity Society; the American Society for Nutrition; and the American Diabetes Association. Am J Clin Nutr. 2007; 85: 1197-1202.

14. Guasch-Ferré M, Bulló M, Martínez-González M, Corella D, Estruch R, Covas $\mathrm{M}$, et al. Waist-to-height ratio and cardiovascular risk factors in elderly individuals at high cardiovascular risk. PLoS One 2012; 7:e43275.

15. Evans P, McIntyre N, Fluck R, McIntyre C, Taal M. Anthropomorphic Measurements That Include Central Fat Distribution Are More Closely Related with Key Risk Factors than BMI in CKD Stage 3. PLoS ONE 2012; 7: e34699.

16. Ashwell M, Gunn P, Gibson S. Waist-to-height ratio is a better screening tool than waist circumference and BMI for adult cardiometabolic risk factors: systematic review and meta-analysis. Obes Rev. 2012; 13: $275-286$.

17. Pinto-Sietsma S, Navis G, Janssen W, de Zeeuw D, Gans R, dr Jong $\mathrm{P}$, et al. A central body fat distribution is related to renal function impairment, even in lean subjects. Am J Kidney Dis. 2003; 41: 733-741.
18. Elsayed E, Tighiouart H, Weiner D, Griffith J, Salem D, Levey A, et al. Waist-to-hip ratio and body mass index as risk factors for cardiovascular events in CKD. Am J Kidney Dis. 2008; 52: 49-57.

19. Kramer H, Shoham D, McClure L, Durazo-Arvizu R, Howard G, Judd $S$, et al. Association of waist circumference and body mass index with all-cause mortality in CKD: The REGARDS (Reasons for Geographic and Racial Differences in Stroke) Study. Am J Kidney Dis. 58: 177-185.

20. Witasp A, Carrero J, Heimburger O, Lindholm B, Hammarqvist $\mathrm{P}$, Nordfors L. Increased expression of pro-inflammatory genes in abdominal subcutaneous fat in advanced chronic kidney disease patients. J Intern Med. 2011; 269: 410-419.

21. Tseng CH. Waist-to-Height Ratio Is Independently and Better Associated With Urinary Albumin Excretion Rate Than Waist Circumference or Waist-to-Hip Ratio in Chinese Adult Type 2 Diabetic Women but Not Men. Diabetes Car. 2005; 28: 2249-2251.

22. Ashwell M, Hsieh S. Six reasons why the waist-to-height ratio is a rapid and effective global indicator for health risks of obesity and how its use could simplify the international public health message on obesity. Int J Food Sci Nutr. 2005; 56: 303-307.

23. Browning L, Hsieh S, Ashwell M. A systematic review of waist-to-height ratio as a screening tool for the prediction of cardiovascular disease and diabetes: 0.5 could be a suitable global boundary value. Nutr Res Rev. 2010; 23: 247-269.

24. Oh H, Quan S, Jeong J, Jang S, Lee J, Kim D. Waist Circumference, Not Body Mass Index, Is Associated with Renal Function Decline in Korean Population: Hallym Aging Study. PLoS ONE 2013; 8: e59071.

25. Cox B, Whichelow M, Ashwell M, Prevost A, Lejeune S. Association of anthropometric indices with elevated blood pressure in British adults. Int J Obes Metab Disord. 1997; 21: 674-680. 